\title{
Clinical and radiographic features of psittacosis infection
}

\author{
II COUTTS, STEPHANIE MACKENZIE, RJ WHITE
}

From Frenchay Hospital, Bristol

ABSTRACT Forty three cases of psittacosis infection were identified retrospectively over a five year period. The commonest clinical presentation was of a bronchitic illness with a productive cough and features of systemic upset. In $12(28 \%)$ cases no radiographic abnormality was detected on the chest film. Segmental shadowing was the commonest abnormality but lobar and more extensive consolidation occurred. There were no radiographic features that allowed confident differentiation from other causes of pneumonia. The total and differential white cell counts were usually normal and this feature may help to differentiate psittacosis from bacterial pneumonia.

Psittacosis is a relatively uncommon cause of community acquired pneumonia in patients requiring hospital admission. In two recent studies of pneumonia, in Bristol ${ }^{1}$ and in Nottingham, ${ }^{2}$ it accounted for only $1.5 \%$ and $5.5 \%$ of cases. In 1980 and 1981 there was an increase in the number of cases of psittacosis detected nationally ${ }^{3}$ and this provided the opportunity to examine a substantial number of cases of psittacosis infection.

\section{Methods}

Patients with psittacosis occurring in the three health districts centred on Bristol in the years 1978-82 were identified from the records of the Public Health Laboratory in Bristol. Our definition of psittacosis infection required a fourfold rise in complement fixing antibody titre or a titre equal to or greater than $1 / 256$ in the absence of positive serological results for other organisms. Most requests for serological testing were made by chest physicians. Determination of antibody titres was usually requested as part of the investigation of pneumonia but was not done routinely in the investigation of symptoms suggesting other acute respiratory tract infections.

The hospital notes and radiographs were obtained for all patients treated either as hospital inpatients

Address for reprint requests: Dr RJ White, Department of General Medicine, Frenchay Hospital, Bristol BS16 1LE.

Accepted 29 January 1985 or as outpatients and the clinical findings were obtained retrospectively from the notes.

The posteroanterior chest radiograph and, where available, a lateral chest film were examined by a physician and a radiologist working together. The extent of the radiographic consolidation was categorised as segmental, lobar, affecting more than one lobe of a lung, or affecting both lungs. The shadowing was described as patchy, confluent, or nodular. Pleural fluid, loss of lung volume, and hilar adenopathy were also recorded. Follow up chest films were examined to determine the rate of resolution of the radiographic changes.

\section{Results}

Forty three patients with psittacosis attended hospital during the five years of the study. Sixteen were managed as inpatients and 27 as outpatients. One patient presented in 1978, four in 1979,16 in 1.980 , 17 in 1981 , and five in 1982 . Twenty four were male and 19 female. The disease was most common during adult working life, with a peak incidence in the fourth decade (table).

The commonest symptom was cough, which occurred in $37(86 \%)$ cases; it was productive of sputum in $28(65 \%)$ cases. In seven cases the sputum was purulent but no organisms were cultured. Some form of systemic upset was very common, $26(60 \%)$ patients reporting febrile symptoms and eight (19\%) headaches. Chest pain occurred in $16(37 \%)$ and was usually pleuritic in type. Less frequent symptoms were breathlessness in seven $(16 \%)$ cases, sore throat or hoarseness in six (14\%), 
Age and sex distribution of cases of psittacosis

\begin{tabular}{llllllllll}
\hline Age $(y)$ & $<20$ & $21-30$ & $31-40$ & $41-50$ & $51-60$ & $61-70$ & $>70$ & Total & Mean (range) \\
\hline Male & 3 & 3 & 6 & 5 & 4 & 2 & 1 & 24 & $41.1(8-76)$ \\
Female & 0 & 4 & 6 & 2 & 5 & 2 & 0 & 19 & $44.1(22-65)$ \\
Total & 3 & 7 & 12 & 7 & 9 & 4 & 1 & 43 & $42.4(8-76)$ \\
\hline
\end{tabular}

haemoptysis in five $(12 \%)$, myalgia in three $(7 \%)$, diarrhoea and vomiting in two, and arthralgia and confusion, each in one case.

Localised inspiratory crackles were found in 23 (53\%) cases but other physical signs were uncommon. Herpes simplex, erythematous rash, erythema nodosum, and hepatomegaly were each recorded once.

The mean white cell count at presentation was $8.15 \times 10^{4}$ (range $\left.3.6 \times 10^{4}-16.2 \times 10^{4}\right) / \mathrm{l}$, with a normal differential count (neutrophils $66 \%$ (range $51-82 \%$ ), lymphocytes $31.5 \%$ (range $17-47 \%$ )).

The mean duration of symptoms before presentation at hospital was 21 days (range 2 days-13 weeks), but symptoms had usually been present for a shorter period in those treated as inpatients (mean 10 days ( 2 days -5 weeks)) than in the outpatients (mean 26 days ( 6 days-13 weeks)).

The chest radiograph was normal at presentation in $12(28 \%)$ cases and in none of these cases did it become abnormal. The mean time from the onset of symptoms to the first chest film was 24 days (range 4 days -13 weeks) in these cases, compared with 20 days ( 2 days -8 weeks) in those with an abnormality.

Radiographic evidence of consolidation occurred in 30 cases. It was patchy in 20 , confluent in eight, and nodular in two. Segmental consolidation was the most frequent abnormality, occurring in $13(31 \%)$ cases. Lobar consolidation occurred in nine $(21 \%)$ cases and consolidation affected more than one lobe in three $(7 \%)$ cases. Both lungs were affected in a further five $(12 \%)$ cases. In only two cases was there good evidence of collapse occurring with the consolidation. Pleural effusion occurred in two cases and in one of these it was the only radiographic abnormality. In the second case it was bilateral and associated with bilateral consolidation. Consolidation was most frequent in the lower lobes. It was present in the right lobe in 11 cases, the left lower lobe in 10 , the middle lobe in four, the lingula in five, the right upper lobe in seven, and the left upper lobe in five. Hilar lymphadenopathy was not observed.

The chest radiograph returned to normal after a mean of 7.2 weeks (range 3-20 weeks) in 19 of the cases with an abnormal film. In nine cases resolution was occurring when the patient was last seen after a mean period of four weeks (range 1-10 weeks); three cases were lost to follow up.

Patients who were admitted to hospital were no more likely than outpatients to have abnormal chest films (12 of $16(75 \%)$ inpatients compared with 19 of $27(70 \%)$ outpatients), but six of the eight cases with consolidation affecting more than one lobe or both lungs were treated as inpatients. The mean ages of the two groups were similar (inpatients 41.5 years (range 17-76); outpatients $42.8(8-65)$ ). The mean duration of symptoms was, however, significantly shorter for patients admitted to hospital (inpatients 10 days (range 2 days -5 weeks), outpatients 26 days ( 6 days -13 weeks)).

As this study was conducted retrospectively we were unable to compile systematic information about exposure to birds, but in many cases subsequent inquiry failed to indicate contact with birds.

\section{Discussion}

Forty three cases of psittacosis infection were identified during 1978-82, 33 of them during 1980 and 1981 at a time when the Public Health Laboratory Service recorded a national increase in cases. Recent studies of community acquired pneumonia and mycoplasma pneumonia ${ }^{4}$ have found a male preponderance but in this series of psittacosis infections and in that of Anderson ${ }^{5}$ there was no sex difference. The disease occurred in all age groups but was most common in the middle decades of life. In contrast to some earlier studies, ${ }^{6}$ the disease was uncommon before the age of 20 .

The most frequently encountered clinical picture was that of a bronchitic illness with a cough, usually productive of sputum, accompanied by systemic symptoms that were often described as flu like Chest pain was common and occurred in more than one third of cases. Physical signs were unimpressive. Crackles were heard in $53 \%$ of cases but other signs were rare.

The chest radiograph was normal in $28 \%$ of cases. Patients with and without radiographic change were seen after a similar interval from the onset of symptoms and, as resolution of the radiographic abnormalities was not particularly rapid, it seems reasonable to conclude that in some cases psittacosis infec- 
tion is not associated with a radiographically apparent pneumonia. These observations point to the occurrence of a psittacosis bronchitis, which should be considered where there is an attack of acute bronchitis with systemic upset, particularly where there is poor response to penicillins or other antibiotics to which chlamydia are insensitive.

The commonest radiographic abnormality was patchy or confluent consolidation, which was most frequently segmental in distribution. Lobar consolidation was seen and in eight cases even more extensive consolidation. Unlike in mycoplasma pneumonia, nodular shadowing was uncommon and, in contrast to Stenström ${ }^{6}$ with his series of ornithosis pneumonia, we did not observe hilar lymphadenopathy. Eleven of Stenström's 29 patients were under 16 years of age; possibly this condition is more common in children. Cavitation has been reported but was not seen in this study.

Radiographic clearance occurred more slowly than in mycoplasma pneumonia, where $96 \%$ of cases had cleared at eight weeks. In this series only half the cases had resolved radiographically at seven weeks, a rate similar to that observed by Macfarlane et $\mathrm{al}^{7}$ in patients with non-bacteraemic, nonantigenaemic pneumococcal pneumonias admitted to hospital.

The mean duration of illness before hospital admission was 10 days, much the same as for mycoplasma pneumonia in Bristol but longer than the mean duration of five days' illness before admission with pneumococcal pneumonia. Outpatients with psittacosis had a much longer history. This difference almost certainly reflects differences in the severity of the infection, very ill patients being admitted to hospital while the less severely ill were referred to clinics. Although the proportions with abnormal radiographs were similar in the inpatient and the outpatient groups, six of the eight patients with consolidation affecting more than one lobe of the lung required hospital admission.

Although extensive consolidation was associated with clinically severe disease, none of the radiographic features allowed discrimination between psittacosis pneumonia and other acute pneumonias. The white cell count, however, was usually within the normal range and this feature may help to exclude a bacterial cause of pneumonia.

\section{References}

1 White RJ, Blainey AD, Harrison JK, Clarke SKR. Causes of pneumonia presenting to a district general hospital. Thorax 1981;36:566-70.

2 Macfarlane JT, Finch RG, Ward MJ, Macrae AD. Hospital study of adult community acquired pneumonia. Lancet 1982;ii:255-8.

3 Palmer SR. Psittacosis in man-recent developments in the UK: a review. J $R$ Soc Med 1982;75:262-7.

4 Finnegan OC, Fowles SJ, White RJ. Radiographic appearances of mycoplasma pneumonia. Thorax 1981;36:469-72.

5 Anderson JP. Ornithosis in Somerset. Experience in the South Somerset clinical area, 1964-71. Postgrad Med J 1973;49:533-4.

6 Stenström R, Jansson E, Wager O. Ornithosis pneumonia with special reference to roentgenological lung findings. Acta Med Scand 1962;171:349-56.

7 Macfarlane JT, Miller AC, Roderick Smith WH, Morris AH, Rose DH. Comparative radiographic features of community acquired legionnaires disease pneumococcal pneumonia, mycoplasma pneumonia and psittacosis. Thorax 1984;39:28-33. 